\section{Peter Galdies}

is Director of DQM Group, a

leading provider of data

governance expertise and solutions. A data governance and marketing professional with over 20 years' experience in the design and development of pragmatic, effective and successful data and data governance information solutions for major corporates, he is a successful business manager involved in coaching and developing other businesses across all business sectors.

Keywords: data security, data management, malware, DQM Group

Majority have suffered data breach

\section{Belief that risk is falling}

DQM Group Limited, DQM House, Baker Street, High Wycombe, HP11 2RX, UK

Tel: +0870 2427788

E-mail: Peter.Galdies@dataiq.co.uk

\section{Business Intelligence}

\section{The insider threat to data assets}

\section{Peter Galdies}

Received (in revised form): 15th January 2014

\begin{abstract}
With the growing importance of data as a driver of business value, security is becoming ever more important. This article looks at recent research carried out into the perception of different types of threat and the level of confidence among organizations in their data security measures.
\end{abstract}

Journal of Direct, Data and Digital Marketing Practice (2014) 15, 197-200. doi:10.1057/dddmp.2014.2

\section{Introduction}

Data is now a critical business asset that delivers value to organizations that capture, manage and develop it responsibly. As a result, the potential for that asset to be stolen and the risks involved in data losses have both increased. Far from being just a worst-case scenario, such problems have been experienced by the majority -54 per cent of organizations surveyed by the Ponemon Institute in 2013 had experienced at least one data breach in the last year, with nearly a fifth experiencing more than four incidents.

At a time when financial penalties for data loss in the United Kingdom now start with the $£ 500,000$ ceiling for fines imposed by the Information Commissioner and rise from there, understanding where vulnerabilities and weaknesses exist in the data security organization is critical.

To this end, research was carried out by DataIQ, part of DQM Group, to understand the level of risk and awareness of that risk that exists among data management and data security professionals. DataIQ is the leading provider of data governance expertise to the marketing industry, specializing in research, auditing and consulting services to both protect and maximize the value of clients' most important asset — their customer data.

\section{Facing up to the risks}

High-profile data losses and breaches, such as that suffered by Sony in 2011, have alerted data owners to the challenges they face. Despite this, confidence in their ability to deal with the issue effectively appears to be high (see Figure 1) - 43.9 per cent of organizations believe the risk is falling, more than double the number who say it is rising (21.2 per cent). Growing maturity in data security measures is clearly supporting this view.

Evidence of just how seriously companies now take the threat can be found in the fact that nearly two-thirds of organizations (65.0 per cent) say that data security is addressed as a board-level issue. In contrast, 
Individuals understand what is required

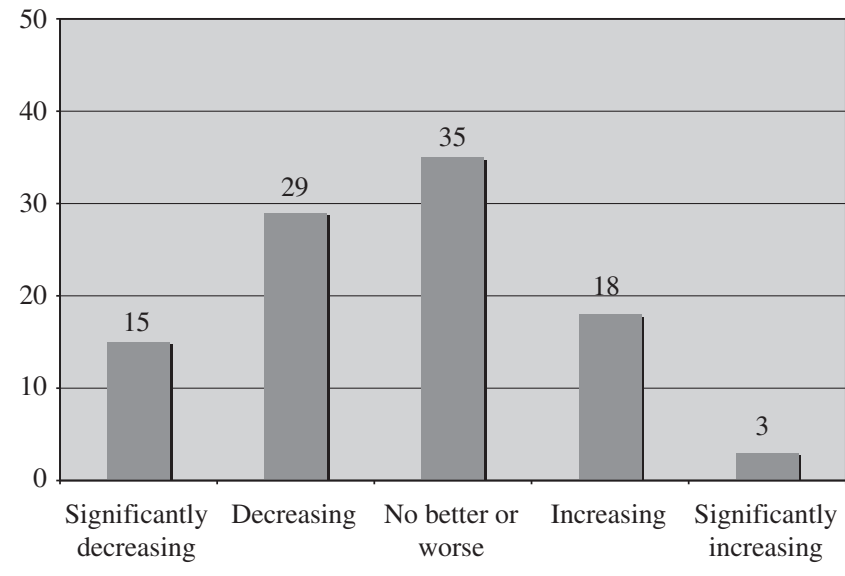

Figure 1: Changing level of data theft risk

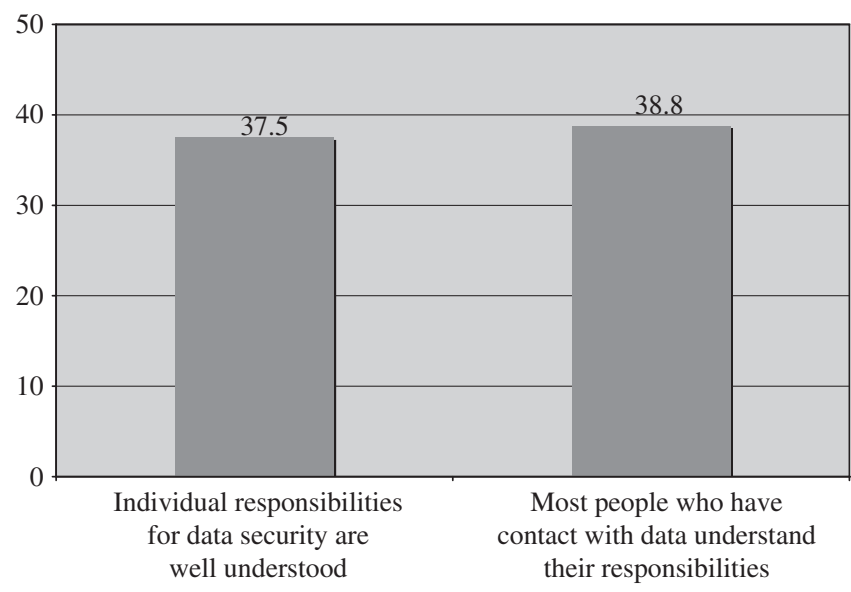

Figure 2: Understanding of data responsibilities

barely a handful accept that their approach is inadequate or that it is not considered important ( 2.6 per cent). In between sits a group of organizations, representing 26.3 per cent of the survey sample, where data security is only taken seriously by limited parts of the business. This seems likely to reflect local data security measures being in place, rather than an over-arching data governance culture.

The basis of effective controls over data has to be ensuring that responsibilities are well understood by those who handle data. On this, three-quarters (76.3 per cent) of organizations take a positive view (see Figure 2), with 37.5 per cent saying individual responsibilities are well understood and 38.8 per cent that most people who have contact with data understand their responsibilities. Again, this leaves around one-quarter of organizations ( 23.8 per cent) exposed by a lack of understanding among staff as to their obligations.

Even so, there are gaps to fill. A bare majority of organizations said that access to data is restricted to only those who need it (52.2 per cent). But 


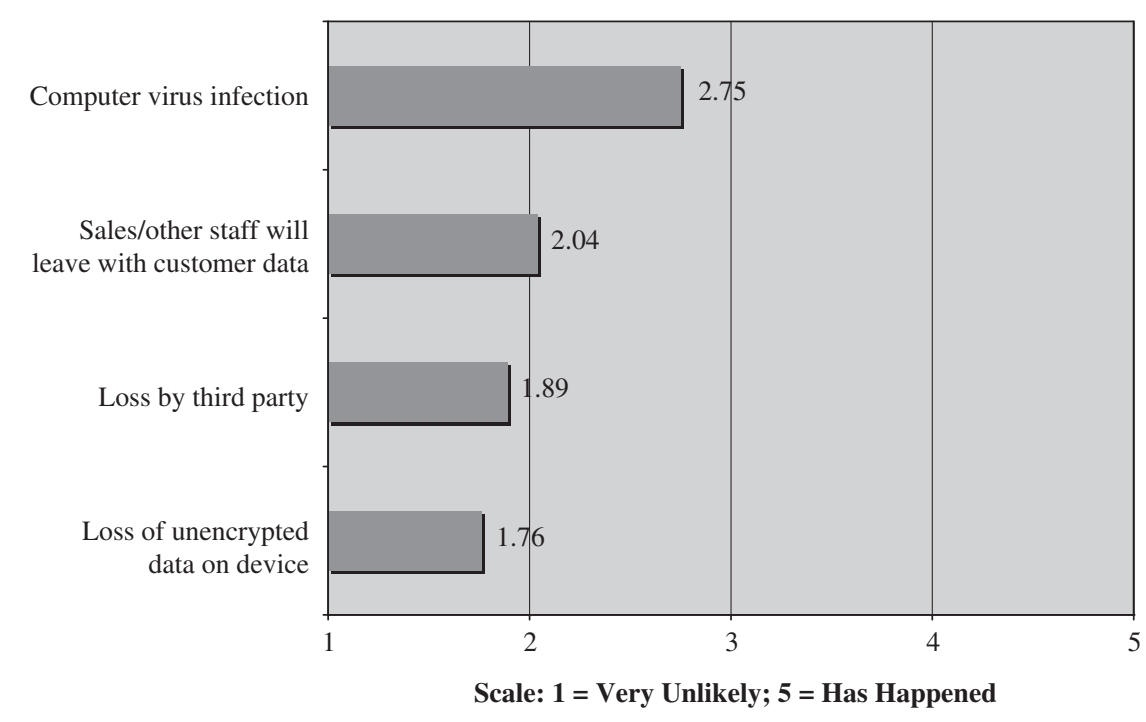

Figure 3: Risks of data loss by type

\section{External threat still most feared}

\section{Insider threat could be under-estimated}

\section{Perception of data loss risks}

Although it is hard to quantify the extent of different data loss risks, some types are more obvious - and therefore more feared - than others. External attacks on the organization that result in a computer virus or malware being installed are perceived as the most likely type of threat to data, although they are still considered unlikely by 79.5 per cent of companies. (See Figure 3 where 5 means such an event has actually happened and 1 means it is considered highly unlikely.) The strong focus on information security measures within IT departments appears to be providing positive reassurance.

Conversely, losing unencrypted data as a result of a device - laptop, tablet or smartphone - being lost by an employee is considered least likely. There was a strongly held view among companies in the sample that this could not happen, perhaps because of confidence in staff being responsible for the technology they use, rather than the fact that they are protective of data. The critical point here, of course, is that data should not be carried unencrypted on any mobile device.

In between, concern about the 'insider threat' - a member of staff leaving the company and taking customer data with them - is also considered unlikely. Some 14.3 per cent of companies even stated that this could not happen, despite it being identified by the UK's fraud prevention 


\section{Best practice solutions can help}

agency, CIFAS, as being 'the most serious challenge for the future'. Indeed, 5.2 per cent admitted that this had happened to them, and recent court cases, such as Flogas v Calor Gas, have highlighted the financial consequences of such thefts.

It is notable that third parties are slightly more trusted than staff members when it comes to keeping data safe. The perceived risk that data will be stolen or lost by an external business partner was ranked second lowest, even though 7.0 per cent had been affected by such an event; by contrast, 14.1 per cent said it could never happen.

\section{Conclusions}

On the basis of their stated perceptions of the risks of different data loss threats, it is clear that organizations believe they have made good strides in countering the problem. Confidence is high that internal procedures and controls are in place to protect data, with individuals who handle it by and large aware of what they need to do.

Nonetheless, gaps remain in the picture of data security. Although the 'insider threat' is less feared than external attacks, this may only be because fewer individual thefts have taken place (or at least become public) compared with attempts to hack a business. In either case, the potential data loss is effectively the same, especially if data is not being routinely encrypted or masked before it is transported and used.

Best practice solutions exist to help reinforce and monitor data management, such as the end-to-end data governance service offered by DQM Group. These not only automate distribution and tracking of data, they also offer legal proof of misuse in the event of a subsequent theft going to trial, which may have a significant impact on any damages that are received.

(The full data security report can be downloaded at http://www .dataiq.co.uk/reports/DATALOSS2013). 